\title{
Implementation of Comparative Probability by States
}

By

\author{
Simba A. Mutangadura*
}

\begin{abstract}
Let $\mathscr{H}$ be an infinite dimensional Hilbert space and $\mathscr{P}(\mathscr{H})$ the set of all (orthogonal) projections on $\mathscr{H}$. A comparative probability on $\mathscr{P}(\mathscr{H})$ is a linear preorder $\preceq$ on $\mathscr{P}(\mathscr{H})$ such that $\mathbf{O} \leq P \leq 1$, $\mathbb{I} \npreceq \mathbf{O}$ and such that if $P \perp R, Q \perp R$, then $P \leq Q \Leftrightarrow P+R \preceq Q+R$ for all $P, Q, R$ in $\mathscr{P}(\mathscr{H})$. In an earlier paper [1], it was shown that weak continuity of $\preceq$ was a sufficient and necessary condition for $\preceq$ to be implemented by a normal state on $\mathscr{B}(\mathscr{H})$, the bounded linear operators on $\mathscr{H}$. In this sequel to [1] we prove that uniform continuity is sufficient and necessary for implementation of $\preceq$ by a state.
\end{abstract}

\section{§. Introduction}

We will generally use the same notation as that of [1], to which this paper is a sequel. Let $\mathscr{H}$ be a Hilbert space and $E$ a (closed) subspace of $\mathscr{H} . \mathscr{P}(E)$ denotes the set of all (orthogonal) projections on $E$ and $P_{E}$ denotes the corresponding projection, with $P_{\phi}$ denoting the projection onto the one dimensional subspace spanned by $\phi$. We drop the $E$ and $\phi$ if no reference to the subspaces is required. $\mathscr{P}_{1}(E)$ is the subset of all one dimensional projections on $E$. Lower case Roman subscripts as in $P_{j}$ or $P_{\phi_{k}}$ will generally be used for indexing sequences and nets. $\mathbb{N}, \mathbb{R}$ and $\mathbb{C}$ denote the natural numbers, the reals and the complex numbers respectively. $P_{\mathscr{H}}$ is denoted by $\mathbb{1}_{\mathscr{H}}$ or just $\mathbb{1}$ if no confusion arises and the zero vector is denoted by $\mathcal{O}$. The orthogonal complement of $P$ (i.e. $1-P$ ) is denoted by $P^{\perp}$. If $P, Q \in \mathscr{P}(\mathscr{H})$ and $P \leq Q^{\perp}$ then we write $P \perp Q$. Finally, we use $P_{j} \stackrel{u}{\rightarrow} P$ and $P_{j} \stackrel{w}{\rightarrow} P$ to mean that the net (or sequence) $P_{j}$ converges to $P$ in the uniform and weak operator topologies respectively.

Definition 1.1. Let $\mathscr{H}$ be any Hilbert space. A preorder relation $\preceq$ on $\mathscr{P}(\mathscr{H})$ is called a comparative probability (CP) iff the following axioms are

Communicated by H. Araki, June 21, 1991

1991 Mathematics Subject Classification: 81P99.

* Dept. of Physics, University of Zimbabwe, Box M.P. 167, Mt Pleasant, Harare, Zimbabwe. 
satisfied by all $P, Q, R \in \mathscr{P}(\mathscr{H})$ :

$$
\begin{array}{ll}
\mathbb{A} 1 & P \leq Q \text { or } Q \leq P, \\
\mathbb{A} 2 & P \leq Q \text { and } Q \leq R \Rightarrow P \leq R, \\
\mathbb{A} 3 & \mathbb{O} \leq P \leq \mathbb{1}, \mathbb{I} \leq \mathbb{O} . \\
\mathbb{A} 4 & \text { If } P \perp R, Q \perp R, \text { then } P \preceq Q \Leftrightarrow P+R \leq Q+R .
\end{array}
$$

We note that axiom $\mathbb{A} A$ is equivalent to the following: If $P \perp R, Q \perp R$, then $P \prec Q \Leftrightarrow P+R \prec Q+R$. Recall that a Gleason measure is a $\sigma$-additive measure on $\mathscr{P}(\mathscr{H})$, that is a $\sigma$-orthoadditive mapping $\mu: \mathscr{P}(\mathscr{H}) \rightarrow[0,1]$ satisfying $\mu(\mathbb{1})=1$. If $\operatorname{dim} \mathscr{H} \neq 2$ and $\mathscr{H}$ remains separable, then Gleason's theorem [3] says that $\mu$ may be extended to a normal state on $\mathscr{B}(\mathscr{H})$. For $\mathscr{H}$ not separable, one trivially verifies that if the $\sigma$-additivity is replaced by complete additivity, then $\mu$ can still be extended to a normal state on $\mathscr{B}(\mathscr{H})$.

More recent work (see [2] for a comprehensive review, including references of the original papers) has generalized Gleason's theorem to include cases where $\mu$ is just an additive measure (i.e. finitely orthoadditive map) acting on the projections of arbitrary Von Neumann algebras. Essentially no new "exceptions" appear beyond the case $\operatorname{dim} \mathscr{H}=2$ which appears in Gleason's theorem. The generalization may be stated as follows: ([2] Theorem 12.1)

Theorem 1.2. Let $\mathscr{A}$ be a Von Neumann algebra without a direct summand of type $\mathbb{I}_{2}$ and let $\mu$ be an additive measure on the projections of $\mathscr{A}$. Then $\mu$ can be extended to a state $\tilde{\mu}$ on $\mathscr{A}$. Further, if $\mu$ is $\sigma$-additive, then $\tilde{\mu}$ is normal if and only if $\mu$ has a support.

As in [1] we wish to establish sufficient and necessary conditions for a $\mathrm{CP} \preceq$ on $\mathscr{P}(\mathscr{H})$, where $\mathscr{H}$ is any infinite dimensional Hilbert space, to be implemented by a state $\omega$ on $\mathscr{B}(\mathscr{H})$ according to the prescription: $\mathbb{P} \preceq Q \Leftrightarrow$ $\omega(P) \leq \omega(Q)$. Where it exists, the implementing state is unique if $\mathscr{H}$ is infinite dimensional but this is generally not true for $\mathscr{H}$ finite dimensional [6], [5]. The proof offered here is also valid for the problem of [1]. Not every $\mathbb{C P}$ can be implemented in this way as the following counter example ${ }^{1}$ shows: Let $\mathscr{H}$ be any Hilbert space of dimension at least three and let $\mathbb{P}_{\phi}$ and $\mathbb{P}_{\psi}$ be mutually orthogonal (one dimensional) projections of $\mathscr{P}(\mathscr{H})$. Define states $\omega_{\phi}$ and $\omega_{\psi}$ on $\mathscr{B}(\mathscr{H})$ by $\omega_{\phi}\left(\mathbb{P}_{\phi}\right)=1$ and $\omega_{\psi}\left(\mathbb{P}_{\psi}\right)=1$. Let $\unlhd$ be defined by $\mathbb{P} \unlhd \mathbb{Q}$ if $\omega_{\phi}(P)<\omega_{\phi}(Q)$ or if $\omega_{\phi}(P)=\omega_{\phi}(Q)$ and $\omega_{\psi}(P) \leq \omega_{\psi}(Q)$. One verifies that $\unlhd$ is indeed a $\mathrm{CP}$ and also that no state can implement it. $\mathbb{A}$ missing crucial ingredient in this $\mathbb{C P}$ is (uniform) continuity. This continuity, unlike the case

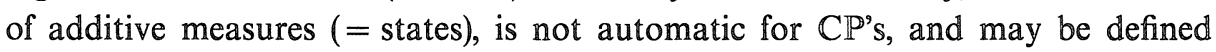
as follows:

\footnotetext{
${ }^{1}$ Communicated to the author by A. Paszkiewicz
} 
Definition 1.3. Let $\mathscr{T}$ be a locally convex topology on $\mathscr{B}(\mathscr{H})$ and $\preceq a$ $\mathrm{CP}$ on $\mathscr{P}(\mathscr{H})$. We say $\preceq$ is $\mathscr{T}$ continuous if whenever a net $P_{j}$ converges to $P$ in the $\mathscr{T}$ topology and $Q \preceq P_{j} \preceq R \quad \forall j$, then $Q \preceq P \preceq R$.

Recall that the $\preceq$ (interval) topology on $\mathscr{P}(\mathscr{H})$ is generated by a neighbourhood base of $\preceq$ intervals of $\mathscr{P}(\mathscr{H})$. For any uniformly continuous CP $\preceq$ on $\mathscr{P}(\mathscr{H})$, addition is separately $\preceq$ continuous (Proposition 2.10 ) in the sense that if $P_{j}$ is a net in $\mathscr{P}(\mathscr{H})$ which $\preceq$ converges to $P \in \mathscr{P}(\mathscr{H})$ (we denote this convergence by $P_{j} \stackrel{\preceq}{\rightarrow}$ ) and there exists $Q \in \mathscr{P}(\mathscr{H})$ such that $P \perp Q$ and $P_{j} \perp Q \forall j$, then $Q+P_{j} \stackrel{\preceq}{\rightarrow} Q+P$. Joint $\preceq$ continuity of addition is harder to establish. We give a formal definition of joint continuity of addition on $\mathscr{P}(\mathscr{H})$ :

Definition 1.4. Addition on $\mathscr{P}(\mathscr{H})$ is said to be jointly $\preceq$ continuous if whenever $P, Q \in \mathscr{P}(\mathscr{H})$ and the nets $P_{j}$ and $Q_{j}$ in $\mathscr{P}(\mathscr{H})$ satisfy $P_{j} \leftrightarrows P, Q_{j} \leqq Q$, $P \perp Q$ and $P_{j} \perp Q_{j} \forall j$, then $P_{j}+Q_{j} \stackrel{\preceq}{\rightarrow} P+Q$.

\section{§2. Uniformly Continuous $\mathbb{C P}^{\prime} \mathbf{s}$}

From now on, $\mathscr{H}$ denotes a complex infinite dimensional Hilbert space which is not necessarily separable, and $\preceq$ denotes a uniformly continuous $\mathbb{C P}$ on $\mathscr{P}(\mathscr{H})$.

Definition 2.1. Let $E$ be a subspace of $\mathscr{H}$. We define $\mathscr{D}(E)$ to be the set of all projections $P_{F} \in \mathscr{P}(E)$ such that the rank of $P_{F}$ and the rank of $\left(P_{E}-P_{F}\right)$ have the same cardinality.

Lemma 2.2. Let $G$ be any infinite dimensional subspace of $\mathscr{H}$. Then $\mathscr{D}(G)$ is uniformly connected.

Proof. Let $P_{E}=\sum_{j \in \Lambda} P_{\phi_{j}}$ and $P_{F}=\sum_{j \in \Lambda} P_{\psi_{j}}$ be both in $\mathscr{D}(G)$ (where the summands in each case are mutually orthogonal) and suppose that $P_{E} \perp P_{F}$. Define the function $f: t \in[0,1] \mapsto \sum_{j \in A} P_{t \phi_{J}+s \psi_{J}} \in \mathscr{D}(G)$, where $s=\sqrt{1-t^{2}}$. One easily shows that $f$ is uniformly continuous. Now suppose $P_{E} \not P_{F}$. There exist projections $P_{E^{\prime}}$ and $P_{F^{\prime}}$, both in $\mathscr{D}(G)$, such that $P_{E^{\prime}} \leq P_{E}$ and $P_{F^{\prime}} \leq P_{F}$ and such that $P_{E^{\prime}} \perp P_{F^{\prime}}$; this can be established, for example, by a simple application of Zorn's lemma. Using the result of the case $P_{E} \perp F_{F}$, we can construct a uniformly continuous path along the route $P_{E} \rightarrow P_{E}^{\perp} \rightarrow P_{E^{\prime}} \rightarrow P_{F^{\prime}} \rightarrow P_{F}^{\perp} \rightarrow P_{F}$.

We remark that the uniform connectedness of $\mathscr{D}(\mathscr{H})$ implies that nets may be replaced by sequences in handling convergence to any projection $Q \in \mathscr{P}(\mathscr{H})$ such that $Q \cong P$ for some $P \in \mathscr{D}(\mathscr{H})$.

Lemma 2.3. Let $P_{E}, P_{F}$ and $P_{K}$, all in $\mathscr{D}(\mathscr{H})$, be mutually orthogonal with $\mathbb{O} \cong P_{E} \prec P_{F}$. Put $P_{G}=P_{K}+P_{F}+P_{E}$. Suppose that $\preceq$ is implemented by a state when restricted to $\mathscr{P}(E+F)$, then there exists a sequence $P_{G}$ in $\mathscr{P}(G)$ 
satisfying the following:

i. $\quad P_{G_{j}} \prec P_{K}+P_{F} \forall j$

迆。 $\quad P_{G_{j}} \stackrel{u}{\rightarrow} P_{K}+P_{F}$

䁌。 If $P_{L}, P_{M} \in \mathscr{P}(F+K)$ are mutually orthogonal, then there exist sequences $S_{j}$ and $T_{j}$ in $\mathscr{P}(G)$ such that $S_{j} \perp T_{j}$ and $S_{j}+T_{j} \leq \mathbb{P}_{G_{j}} \forall j$, and such that $S_{j} \stackrel{u}{\rightarrow} P_{L}, \quad T_{j} \stackrel{u}{\rightarrow} P_{M}$.

Proof. Let $P_{E}=\sum_{j \in \Lambda} P_{\phi_{j}}$ and $P_{F}=\sum_{j \in \Lambda} P_{\psi_{j}}$. For each $t \in[0,1]$, define the function $f_{t}: K+E \rightarrow G$ by $f_{t}\left(\phi_{j}\right)=s \phi_{j}+t \psi_{j} \forall j \in \Lambda$ and $f_{t}(\xi)=\xi \forall \xi \in \mathbb{K}$, where $s=\sqrt{1-t^{2}}$. Then the restriction of $f_{t}$ to any subspace $J$ of $K+E$ is a unitary operator onto $f_{t}(J)$ and the map $t \mapsto P_{f_{t}(J)}$ is uniformly and hence $\preceq$ continuous. Thus there exists a sequence of the form $P_{f_{t_{j}}(E)}$ such that $P_{f_{t_{j}}(E)} \stackrel{u}{\rightarrow}$ $P_{F}$ and $P_{f_{t}(E)} \preceq P_{F} \forall j$. But for any $r$ such that $0<r<1, P_{E}$ is in the linear span of the set $\left\{\mathbb{P}_{f_{t}(E)}: r \leq t \leq 1\right\} \subset \mathscr{P}(E+F)$. Since $\preceq$ is implemented by a state on $\mathscr{P}(E+F)$, we cannot have $P_{f_{t}(E)} \cong \mathbb{P}_{F} \forall t \geq r$. Thus the $t_{j}$ may be chosen so as to give the strict inequality $\mathbb{P}_{f_{t_{j}}(E)} \prec P_{F} \forall j$ so that the sequence $P_{f_{t_{j}}(K+E)}$ satisfies items (i) and (ii).

To show that this sequence also satisfies item (iii) we put $\tilde{L}=f_{1}^{-1}(L)$ and $\tilde{M}=f_{1}^{-1}(M)$. Then the sequences $P_{f_{t_{j}}(\tilde{L})}$ and $P_{f_{t_{j}}(\tilde{M})}$ will satisfy the requirements of item (iii).

Proposition 2.4. The following are both true:

I. Let $P_{G} \in \mathscr{D}(\mathscr{H})$ and let $P \in \mathscr{P}(\mathscr{H})$ be such that $\mathbb{O}<\mathbb{P}$. Then there exists $Q \in \mathscr{D}(G)$ such that $Q \preceq P$.

ii. Let $\mathscr{A} \subset \mathscr{D}(\mathscr{H})$ be a set of mutually orthogonal projections. Suppose that there exists $R \in \mathscr{P}(\mathscr{H})$ such that $\mathbb{O}<R$ and such that $\mathbb{R}^{\prime} \in \mathscr{A} \Rightarrow \mathbb{R} \preceq R^{\prime}$, then $\mathscr{A}$ is a finite set.

Proof. ․ First we claim that there exists $Q \in \mathscr{D}(\mathscr{H})$ such that $Q \preceq \mathbb{P}$. Suppose that this is false. Then there exists $P_{E} \in \mathscr{P}(\mathscr{H})$ such that $P_{E} \leq P_{E^{\prime}}$ for some $P_{E^{\prime}} \in \mathscr{D}(\mathscr{H})$ and such that $\mathscr{O}<P_{E} \preceq R \forall R \in \mathscr{D}(\mathscr{H})$. Now we can clearly construct two sequences $P_{j}$ and $Q_{j}$, both in $\mathscr{D}\left(E^{\perp}\right)$, such that $P_{j} \perp P_{k}, Q_{j} \perp Q_{k}$ $\forall j, k \in \mathbb{N}$ with $j \neq k$, and such that $P_{j} \perp Q_{k}, P_{j} \cong Q_{j} \forall j, k \in \mathbb{N}$. Let $P_{F}=\sum_{j=1}^{\infty} P_{j}$ and define $S_{n}$ to be $P_{E}+\sum_{j=2}^{n} P_{j}$. Clearly, $S_{n} \in \mathscr{D}(\mathscr{H}) \forall n$ and since $\mathcal{O} \prec P_{E}, S_{n}$ is a strictly $\preceq$ increasing sequence. As $\mathscr{D}(F)$, and hence also $P_{E}+\mathscr{D}(F)$, are uniformly connected, there exists $S \in \mathscr{D}(F)$ such that $S_{n} \stackrel{\preceq}{\rightarrow} \mathbb{P}_{E}+S$. Thus for some $n_{0} \in \mathbb{N}, S \prec S_{n_{0}}$, so that by $\mathbb{A} 4, S+P_{E} \preceq S+Q_{n_{0}+1} \prec S_{n_{0}}+Q_{n_{0}+1} \cong S_{n_{0}+1}$. The contradiction verifies the claim.

Now suppose that the assertion in (i) is false. Then there exists $\mathbb{R} \in \mathscr{P}(\mathscr{H})$ such that $\mathscr{O}<R \preceq Q \forall Q \in \mathscr{D}(G)$. The claim above implies that there exists 
$P_{L} \in \mathscr{D}(\mathscr{H})$ such that $P_{L} \prec R$. As pointed out in Lemma 2.2, there exist $P_{E} \in$ $\mathscr{D}(L)$ and $P_{F} \in \mathscr{D}(G)$ such that $P_{E} \perp P_{F}$. Let $P_{K} \in \mathscr{D}(F)$ be such that $P_{K} \prec P_{F}-$ $P_{K}$ and let $P_{E_{1}}, P_{E_{2}} \in \mathscr{D}(E)$ be such that $P_{E_{1}} \preceq P_{E_{2}}$. Then $\mathbb{A} 4$ gives $P_{K}+P_{E_{1}} \prec$ $P_{F}+P_{E_{2}}-P_{K}$. Since $\mathscr{D}\left(K+E_{1}\right)$ is $\preceq$ connected and $P_{E_{1}} \in \mathscr{D}\left(K+E_{1}\right)$, there exists $S \in \mathscr{D}\left(K+E_{1}\right)$ such that $S \in \preceq$ inf $\mathscr{D}(K)$. As $P_{E_{2}} \perp\left(P_{K}+P_{E_{1}}\right), \quad \exists T \in$ $\mathscr{D}\left(\left\{K+E_{1}\right\}^{\perp}\right)$ such that $O \prec T \prec R$. Let the sequence $S_{j}$ in $\mathscr{D}(K)$ be $\preceq$ convergent to $S$. For each $j \in \mathbb{N}$, let $S_{j}^{\prime} \leq S_{j}$ be such that $S_{j}^{\prime} \cong S_{j}-S_{j}^{\prime}$. Then $T+S \preceq T+\left(S_{j}-S_{j}^{\prime}\right) \prec S_{j}^{\prime}+\left(S_{j}-S_{j}^{\prime}\right) \rightarrow S$, a contradiction.

ii. Suppose that the statement is not true, so that $\mathscr{A}$ is an infinite set. By taking a subset if necessary, we may assume that $\mathscr{A}=\left\{Q_{j}: j \in \mathbb{N}\right\}$, that is $\mathscr{A}$ is countable. We may also assume that $\sum_{j \in \mathbb{N}} Q_{j} \leq Q-\sum_{j \in \mathbb{N}} Q_{j}$, for some $Q \in \mathscr{D}(\mathscr{H})$ such that $\sum_{j \in \mathrm{N}} Q_{j}<Q$. Define $P_{n}$ to be $\sum_{j=n}^{\infty} Q_{j}$. Then $P_{n}$ is a strictly $\preceq$ decreasing sequence. Now $\forall n \in \mathbf{N}, R \prec P_{n}$ and $P_{n} \in \mathscr{D}(\mathscr{H})$; thus, by (i) and by the uniform connectedness of $\mathscr{D}(\mathscr{H})$, there exists $P_{0} \in \mathscr{D}(\mathscr{H})$ such that $P_{0} \in$ $\preceq \inf _{n \in \mathbf{N}} P_{n}$. Put $Q-\sum_{j \in \mathbb{N}} Q_{j}=P_{F}$ and $\mathbb{1}-\sum_{j \in \mathbb{N}} Q_{j}=P_{K}$. Then $P_{0} \preceq P_{F}$ and $P_{F} \in$ $\mathscr{D}(K)$. Hence, by (i) and by the connectedness of $\mathscr{D}(K)$, we may assume that $P_{0} \in \mathscr{D}(K)$. Let $R^{\prime} \in \mathscr{D}(K)$ be such that $R^{\prime} \perp P_{0}$ and $O \prec R^{\prime} \preceq R$. Then, for some large enough $n, P_{n} \prec P_{0}+R^{\prime}$ and for such $n, P_{n}=P_{n+1}+Q_{n} \prec P_{0}+R^{\prime} \preceq$ $P_{0}+Q_{n} \Rightarrow P_{n+1} \prec P_{0}$, by $\mathbf{A} 4$. The contradiction gives the required result.

Corollary 2.5. The following are both true: $P_{j} \stackrel{0}{\rightarrow}$.

i. If $P_{j}$ is a sequence of mutually orthogonal projections of $\mathscr{P}(\mathscr{H})$, then

ii. $\mathscr{P}(E)$ is $\preceq$ connected if $P_{E} \in \mathscr{D}(\mathscr{H})$.

Proof. i. Clear.

ii. In view of Proposition 2.4 (i), it is sufficient to show that $P_{E}$ is a $\preceq$ limit point of $\mathscr{D}(E)$. If $P_{E} \cong \mathbf{O}$ or $P_{E} \cong \mathbb{I}$ then this is trivial, so assume that $\mathcal{O} \prec P_{E} \prec \mathbb{1}$ and suppose that the result is false. Then there exists $Q \in \mathscr{P}(E)$ such that $O \prec Q$ and such that $P \preceq P_{E}-Q \forall P \in \mathscr{D}(E)$. Now there exist $S \in$ $\mathscr{D}(E)$ and $T \in \mathscr{D}\left(E^{\perp}\right)$ such that $S \cong T \prec Q$. $\mathbb{A} 4$ gives $P_{E}-Q+T \prec P_{E}=P_{E}-$ $S+S \cong P_{E}-S+T \Rightarrow P \preceq P_{E}-Q \prec P_{E}-S \forall P \in \mathscr{D}(E)$, a contradiction, since $P_{E}-S \in \mathscr{D}(E)$.

Lemma 2.6. Let $P_{E} \in \mathscr{D}(\mathscr{H})$ be such that $P_{E} \preceq P_{E}^{\perp}$ and let $P_{1}, P_{2}, Q_{1}$ and $Q_{2}$, all in $\mathscr{P}(E)$, be such that $P_{1} \perp Q_{1}, P_{2} \perp Q_{2}, P_{1} \preceq P_{2}$ and $Q_{1} \preceq Q_{2}$. Then $P_{1}+Q_{1} \preceq P_{2}+Q_{2}$. Hence for any $R_{1}, R_{2} \in \mathscr{P}(E)$ and $Q \in \mathscr{P}(\mathscr{H})$ such that $R_{1} \leq Q$ and $R_{2} \leq Q$ we have $R_{1} \preceq R_{2} \Leftrightarrow Q-R_{2} \preceq Q-R_{1}$.

Proof. Since $P_{E}^{\perp} \in \mathscr{D}(\mathscr{H})$ and $P_{E} \preceq P_{E}^{\perp}$, we can find, using the $\preceq$ connectedness of $\mathscr{P}\left(E^{\perp}\right)$ and the continuity of $\preceq$ on $\mathscr{P}(\mathscr{H})$ (and hence on $\mathscr{P}\left(E^{\perp}\right)$ ), 
$R \leq P_{E}^{\perp}$ such that $P_{1} \preceq R \preceq P_{2}$. This gives, by axiom $\mathbb{A} 4 P_{1}+Q_{1} \preceq R+Q_{1} \preceq$ $R+Q_{2} \preceq P_{2}+Q_{2}$, as required. We note that this result implies the following: if $P \leq P_{E}$ and $Q \leq P_{E}$, then $P \preceq Q \Leftrightarrow P_{E}-Q \preceq P_{E}-P$. To prove the second part we let $R=R_{1} \vee R_{2}$ (the minimal projection in $\mathscr{P}(\mathscr{H})$ containing $R_{1}$ and $R_{2}$ as sub-projections). As $R \preceq R^{\perp}$, the first result gives $\mathbb{R}_{1} \preceq R_{2} \Leftrightarrow \mathbb{R}-\mathbb{R}_{2} \preceq$ $R-R_{1}$. Axiom $\mathbb{A}$ gives $R-R_{2} \preceq R-R_{1} \Leftrightarrow Q-R_{2} \preceq Q-\mathbb{R}_{1}$, giving the required result.

Proposition 2.7. $\mathscr{P}(\mathscr{H})$ is $\preceq$ connected.

Proof. It will suffice to show that $\mathbb{l} \preceq \preceq \sup \mathscr{D}(\mathscr{H})$. Now suppose that

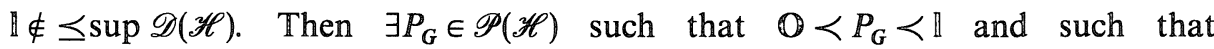
$Q \preceq P_{G}^{\perp} \forall Q \in \mathscr{D}(\mathscr{H})$. We examine two cases:

Case $1 \mathscr{D}\left(G^{\perp}\right)$ is $\preceq$ dense in $\mathscr{P}\left(G^{\perp}\right)$.

Clearly $P_{G} \notin \mathscr{D}(\mathscr{H})$, being of too small a rank. Take $Q \in \mathscr{D}\left(G^{\perp}\right)$, then $P_{G} \preceq Q^{\perp}$. As $\mathscr{D}\left(G^{\perp}\right)$ is $\preceq$ dense in $\mathscr{P}\left(G^{\perp}\right)$ and $\preceq$ connected, $\exists \mathbb{P} \in \mathscr{D}\left(G^{\perp}\right)$ such that $P \cong Q^{\perp}$. Let $R_{1}, R_{2} \in \mathscr{D}\left(G^{\perp}\right)$ be such that $R_{1} \perp R_{2},\left(R_{1}+R_{2}\right) \perp P, R_{1} \preceq$ $R_{2}$ and $R_{1} \prec P_{G}$. Axiom $\mathbb{A} 4$ gives $R_{1}+P_{G} \preceq R_{1}+P \preceq \mathbb{P}+R_{2}$. Since $R_{1}+$ $P_{G} \perp P+R_{2}$, we have $R_{1}+P_{G} \preceq\left(R_{1}+P_{G}\right)^{\perp}$ so that, by Lemma 2.6 , $1-P_{G} \prec$ $\mathbb{1}-R_{1}$, a contradiction.

Case $2 \mathscr{D}\left(G^{\perp}\right)$ is not $\preceq$ dense in $\mathscr{P}\left(G^{\perp}\right)$.

This implies that $\mathbb{O} \prec P_{G}$ by Corollary 2.5 (ii) and that there exists $P_{K} \in$ $\mathscr{P}\left(G^{\perp}\right)$ such that $\mathbb{O} \prec P_{K} \prec \mathbb{P}_{G}^{\perp}, P_{K} \preceq P_{G}$ and such that $Q \preceq P_{G}^{\perp}-P_{K} \forall Q \in \mathscr{D}\left(G^{\perp}\right)$. Put $P_{J}=P_{K}+P_{G}$. Then $\exists S, T \in \mathscr{D}\left(J^{\perp}\right)$ such that $S \perp \mathbb{T}, S \prec P_{K}$, and $S \preceq T$. Axiom $\mathbb{A} 4$ gives $S+P_{K} \preceq S+P_{G} \preceq T+P_{G} \Rightarrow S+P_{K} \preceq\left(S+P_{K}\right)^{\perp}$ so that, by Lemma 2.6, $P_{G}^{\perp}-P_{K} \prec \mathbb{P}_{G}^{\perp}-S \in \mathscr{D}\left(G^{\perp}\right)$, a contradiction. This completes the proof.

We remark that, by Lemma 2.2 and Proposition 2.7, if $G$ is any infinite dimensional subspace of $\mathscr{H}$, then $\mathscr{D}(G)$, and hence also $\mathscr{P}(G)$, are $\preceq$ connected in $\mathscr{P}(\mathscr{H})$. The following results, which we list without proof, are easy consequences of the foregoing results (cf. [1], Theorem 2.3).

Theorem 2.8. The following statements are all true:

i. $\mathscr{P}(\mathscr{H})$ is $\preceq$ compact and hence every nonempty subset of $\mathscr{P}(\mathscr{H})$ has an inf and a sup with respect to $\preceq$.

i月. Let $\mathscr{A} \subset \mathscr{P}(\mathscr{H})$ be any set of mutually orthogonal projections such that $\forall P \in \mathscr{A}, \mathbb{O} \prec P$. Then $\mathscr{A}$ is at most a countably infinite set.

Joint $\preceq$ continuity on $\mathscr{P}(\mathscr{H})$ is essentially a strengthening of axiom $\mathbb{A} 4$.

Proposition 2.9. The following statements are equivalent.

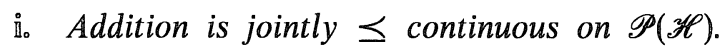


ii. Let $P_{1}, P_{2}, Q_{1}$ and $Q_{2}$, all in $\mathscr{P}(\mathscr{H})$, be such that $P_{1} \perp Q_{1}, P_{2} \perp Q_{2}$, $P_{1} \preceq P_{2}$ and $Q_{1} \preceq Q_{2}$, then $P_{1}+Q_{1} \preceq P_{2}+Q_{2}$

Proof. i. $\Rightarrow$ ii. First we remark that the joint $\preceq$ continuity of addition immediately gives $P_{1}+Q_{1} \cong P_{2}+Q_{2}$ if $P_{1} \cong P_{2}$ and $Q_{1} \cong Q_{2}$. We shall assume that $P_{2}+Q_{2} \prec \mathbb{I}$ and that $\mathbb{O} \prec P_{1}, \mathbf{O} \prec Q_{1}, \mathbf{O} \prec P_{2}, \mathbf{O} \prec Q_{2}$, otherwise the result is trivially true. Assume, with no loss of generality, that $R \leq Q_{2}^{\perp}$ for some $R \in \mathscr{D}(\mathscr{H})$. Since $O \prec P_{2} \prec Q_{2}^{\perp}$, there exist by Proposition 2.7, $P_{1}^{\prime}$, $P_{2}^{\prime} \in \mathscr{D}(\mathscr{H})$ such that $P_{1}^{\prime} \leq P_{2}^{\prime} \leq Q_{2}^{\perp}, P_{1}^{\prime} \cong P_{1}$ and $P_{2}^{\prime} \cong P_{2}$. Hence there similarly exist $Q_{1}^{\prime}, Q_{2}^{\prime} \in \mathscr{D}(\mathscr{H})$ such that $Q_{1}^{\prime} \leq Q_{2}^{\prime} \leq P_{2}^{\prime \perp}, Q_{1}^{\prime} \cong Q_{1}$ and $Q_{2}^{\prime} \cong Q_{2}$. Thus $P_{1}+Q_{1} \cong P_{1}^{\prime}+Q_{1}^{\prime} \preceq P_{2}^{\prime}+Q_{2}^{\prime} \cong P_{2}+Q_{2}$, as required.

$$
\text { ii. } \Rightarrow \text { i. }
$$

Let $P, Q$ and the nets $P_{j}, Q_{j}: j \in \mathscr{J}$, all in $\mathscr{P}(\mathscr{H})$, be such that $P_{j} \stackrel{\Im}{\rightarrow} P$, $Q_{j} \stackrel{\preceq}{\rightarrow}, P \perp Q$ and $P_{j} \perp Q_{j} \forall j$. First we consider the case where both $P_{j}$ and $Q_{j}$ are monotone $\preceq$ increasing and assume that $O \prec P$ and $O \prec Q$, lest the result be trivial. Hence we may also assume that $P, Q \in \mathscr{D}(\mathscr{H})$. Now item (ii) implies that $P_{j}+Q_{j}$ is monotone $\preceq$ increasing and hence $\preceq$ convergent to $R \in \preceq \sup _{j}\left(P_{j}+Q_{j}\right)$. Clearly $R \preceq P+Q$. Suppose that $R \prec P+Q$. Then, by hypothesis, there exist $\tilde{P}<P$ and $\tilde{Q}<Q$ such that $R \prec \tilde{P}+\tilde{Q} \prec P+Q$. This implies that for all $j$ large enough, we have $P_{j}+Q_{j} \prec \tilde{P}+\tilde{Q}, \tilde{P} \prec P_{j} \prec P$ and $\tilde{Q} \prec Q_{j} \prec Q$. The contradiction gives $R \cong P+Q$. For the case where both $P_{j}$ and $Q_{j}$ are monotone $\preceq$ decreasing a similar strategy gives the same result.

Now we consider the case where $P_{j}$ is monotone $\preceq$ increasing and $Q_{j}$ is monotone $\preceq$ decreasing and consider only the case $O \prec P$, otherwise the result is trivially true. Hence we may assume here that $P$ is of infinite rank. Now there exists, by Theorem 2.8, a subnet $P_{j_{k}}+Q_{j_{k}}$ of $P_{j}+Q_{j}$ which is $\preceq$ convergent to $R$, say. We claim that $R \cong P+Q$. To show this we suppose first that $R \prec P+Q$. As $P$ is of infinite rank, there exists $\tilde{P}<P$ such that $\tilde{P}^{\perp}$ is of infinite rank and such that $R \prec \tilde{P}+Q \prec P+Q$. This in turn implies that there exists $\tilde{Q}<\tilde{P}^{\perp}$ such that $R \prec \tilde{P}+Q \prec \tilde{P}+\tilde{Q} \prec P+Q$. Hence there exists $j_{0} \in \mathscr{J}$ such that $\forall j_{k}>j_{0}, P_{j_{k}}+Q_{j_{k}} \prec \tilde{P}+Q$ and $\widetilde{P} \prec P_{j_{k}} \prec P, Q \prec Q_{j_{k}} \prec \tilde{Q}$. The last two inequalities give, by hypothesis, $\tilde{P}+Q \prec P_{j_{k}}+Q_{j_{k}} \forall j_{k}>j_{0}$, a contradiction. A similar argument establishes that we cannot have $P+Q \prec R$, and the claim is verified. Finally, we note that for the general case, an easy argument involving subnets of $P_{j}+Q_{j}$ completes the proof.

Proposition 2.10. Addition is separately $\preceq$ continuous on $\mathscr{P}(\mathscr{H})$.

Proof. Let $P, P_{G}$ and the net $P_{j}: j \in \mathscr{J}$, all in $\mathscr{P}(\mathscr{H})$, be such that $P_{j} \lesseqgtr P$, $P \perp P_{G}$ and $P_{j} \perp P_{G} \forall j$. If $P_{G}^{\perp}$ is of finite rank, then every subnet of $P_{G}+P_{j}$ has itself a subnet which uniformly converges to a projection of the form 
$P_{G}+P^{\prime}$ where $P^{\prime} \perp P_{G}$ and, by the uniform continuity of $\preceq, \mathbb{P}^{\prime} \cong \mathbb{P}$, hence the result. For $P_{G}^{\perp}$ of infinite rank, we assume for the moment that $P_{j}$ is monotone $\preceq$ increasing, and hence $\preceq$ convergent to $R$, say. Clearly $R \preceq P+\mathbb{P}_{G}$. If $R \prec P+P_{G}$, then, since $\mathscr{D}\left(G^{\perp}\right)$ and hence $P_{G}+\mathscr{D}\left(G^{\perp}\right)$ are $\leq$ connected, there exists $P^{\prime} \in \mathscr{D}\left(G^{\perp}\right)$ such that, for all $j, P_{G}+P_{j} \preceq P_{G}+P^{\prime} \prec P_{G}+P$, contradicting $P_{j} \leftrightarrows P$. Hence $P_{G}+P_{j} \leftrightarrows P_{G}+P$. A similar argument gives the same result if we assume $P_{j}$ to be monotone $\preceq$ decreasing. For $P_{j}$ arbitrary, we note that every subnet of $P_{j}$ has a subnet that $\preceq$ converges to $\mathbb{P}_{G}+\mathbb{P}$, and the result follows.

Corollary 2.11. $\mathscr{P}(\mathscr{H})$ is $\preceq$ first countable.

Proof. Let $P_{E} \in \mathscr{D}(\mathscr{H})$ be such that $O<P_{E} \preceq P_{E}^{\perp}$ and let the sequence $Q_{j}$ in $\mathscr{D}(E)$ be such that $\mathscr{O} \prec Q_{j} \forall j$ and $Q_{j} \leftrightarrows \mathbb{O}$. Then Lemma 2.6 and Corollary 2.5 (ii) imply that $P_{E}-Q_{j} \stackrel{\preceq}{\rightarrow} P_{E}$ and Proposition 2.10 gives $P_{E}^{\perp}+P_{E}-Q_{j} \leftrightarrows \mathbb{1}$. Put $P_{E}^{\perp}+P_{E}-Q_{j}=P_{j}$ then the two sets of $\preceq$ intervals $\left[0, Q_{j}\right): j \in \mathbb{N}$ and $\left(P_{j}, \mathbb{1}: j \in \mathbb{N}\right.$ form countable $\preceq$ neighbourhood bases for $\mathbb{O}$ and $\mathbb{1}$ respectively. The remark at the end of Lemma 2.2 completes the proof.

\section{§3. Implementability of Continuous $\mathbb{C P}^{9} \mathrm{~s}$}

In this section, we prove a number of results which will culminate in the construction of an additive measure on $\mathscr{P}(\mathscr{H})$. This measure will, by Theorem 1.2, lead to a unique state which implements $\preceq$.

Lemma 3.1. For $n \in \mathbb{N}$, let the sets $\left\{P_{j}: 1 \leq j \leq n\right\}$ and $\left\{Q_{j}: 1 \leq j \leq n\right\}$, each of which consists of mutually orthogonal projections in $\mathscr{P}(\mathscr{H})$, be such that $P_{j} \perp Q_{k} \forall j, k$ and such that for each $j, P_{j} \preceq Q_{j}$. Then $\sum_{j=1}^{n} P_{j} \preceq \sum_{j=1}^{n} Q_{j}$.

Proof. Let $m \in \mathbb{N}$ be such that $m<n$ and suppose that $\sum_{j=1}^{m} P_{j} \preceq \sum_{j=1}^{m} Q_{j}$. Axiom A4 gives $\sum_{j=1}^{m+1} P_{j}=P_{m+1}+\sum_{j=1}^{m} P_{j} \preceq P_{m+1}+\sum_{j=1}^{m} Q_{j} \preceq Q_{m+1}+\sum_{j=1}^{m} Q_{j}=\sum_{j=1}^{m+1} Q_{j}$. Since $P_{1} \preceq Q_{1}$ the result follows by induction.

Lemma 3.2. Given any $n \in \mathbb{N}$, there exists a set $\left\{P_{E_{j}}: j \in K(n)\right\}$, where $K(n)=$ $\left\{1,2,3, \ldots, 2^{n}\right\}$, of mutually orthogonal projections such that $P_{E_{j}} \in \mathscr{D}(\mathscr{H}) \forall j, P_{E_{j}} \cong$ $P_{E_{k}} \forall j, k$ and such that $\sum_{j \in K(n)} P_{E_{j}}=\mathbb{I}$.

Proof. Suppose the result to be true for some $n_{0} \in \mathbb{N}$. For each $j \in K\left(n_{0}\right)$, $\preceq$ is obviously a uniformly continuous $\mathrm{CP}$ when restricted to $\mathscr{P}\left(E_{j}\right)$ and because $\mathscr{D}\left(E_{j}\right)$ is $\preceq$ connected and $\preceq$ dense in $\mathscr{P}\left(E_{j}\right)$, there exists $P_{\tilde{E}_{j}} \in \mathscr{D}\left(E_{j}\right)(\subset \mathscr{D}(\mathscr{H}))$ such that $P_{E_{j}}-P_{\widetilde{E}_{j}} \cong P_{\tilde{E}_{j}}$. This, together with Lemma 3.1, is enough to show 
that the result is also true for both $n=1$ and $n=n_{0}+1$ and hence, by induction, for all $n \in \mathbb{N}$.

Definition 3.3. A set $\left\{P_{E_{j}}: j \in K(n)\right\}$ satisfying the assertion of Proposition 3.2 is said to be an equipartition of the identity of order $2^{n}$. Let $\mathscr{L}=$ $\left\{P_{E_{J}}: j \in K(n)\right\}$ be an equipartition of the identity of order $2^{n}$, we define $\mathscr{S}(\mathscr{L}) \subset$ $\mathscr{D}(\mathscr{H})$ to be the set $\left\{\sum_{j \in K} P_{E_{j}}: K \subset K(n), K \neq \varnothing, K \neq K(n)\right\}$. It is clear that there exists a sequence of equipartitions $\mathscr{L}_{n}$ of the identity such that $\mathscr{L}_{n}$ is of order $2^{n}$ and such that the $\mathscr{L}_{n}$ are "nested" in the sense that $\mathscr{S}\left(\mathscr{L}_{n}\right) \subset$ $\mathscr{S}\left(\mathscr{L}_{n+1}\right) \forall n \in \mathbb{N}$. When there is such nesting, we define $\mathscr{S}_{\infty}$ to be $\bigcup_{n \in \mathbf{N}} \mathscr{S}\left(\mathscr{L}_{n}\right)$.

From now on we work with a fixed set of nested equipartitions $\mathscr{L}_{n}$ as set out in Definition 3.3.

Proposition 3.4. $\mathscr{S}_{\infty}$ is $\preceq$ dense in $\mathscr{P}(\mathscr{H})$.

Proof. Assuming the axiom of choice, let $P_{j} \in \mathscr{L}_{j} \forall j \in \mathbb{N}$. Clearly $P_{j} \leftrightarrows 0$ and $P_{j}^{\perp}\left(\in \mathscr{L}_{j}\right) \stackrel{\preceq}{\rightarrow}$. Now let $P \in \mathscr{P}(\mathscr{H})$ be such that $\mathbb{O} \prec P \prec 1$. Then, because $0<P^{\perp}$, there exists, for some $n \in \mathbb{N}, R \in \mathscr{L}_{n}$ such that $R \prec P$. We may assume, without loss of generality, that $R \perp P$. We wish to show that $P$ is a $\preceq$ limit point of $\mathscr{S}_{\infty}$. Consider the set $\Gamma=\left\{Q \in \mathscr{S}_{\infty}: Q \preceq P\right\}$. Let $Q_{0} \in \preceq \sup \Gamma$. As $Q_{0} \leq P \leq R^{\perp}$, we may also assume that $Q_{0} \perp R$. Because of the nesting of the $\mathscr{L}_{j}$ and because $Q_{0} \leq R^{\perp}$, we have $Q_{0} \in \preceq$ sup $\tilde{\Gamma}$ where $\tilde{\Gamma}=\left\{Q \in \mathscr{S}_{\infty}: Q \preceq P\right.$, $\left.Q \leq R^{\perp}\right\}$. We now show that $Q_{0} \cong P$. Suppose this is false, so that $Q_{0} \prec P$. Then there exists $R_{0} \in \mathscr{L}_{m}$ for some $m$ such that $R_{0} \leq R$ and such that $Q_{0}+R_{0} \prec P$. Now let the sequence $Q_{j}$ in $\tilde{\Gamma} \preceq$ converge to $Q_{0}$. Then there clearly exists $n$ such that $Q_{0} \prec Q_{n}+R_{0}$. As $Q_{j} \preceq Q_{0} \forall j$, A4 gives $Q_{0} \prec Q_{n}+$ $R_{0} \preceq Q_{0}+R_{0} \prec P$. Since $Q_{n}+R_{0} \in \mathscr{S}_{\infty}$, this contradicts $Q_{0} \in \preceq \sup \Gamma$; hence the result.

Definition 3.5. Define the function $\mu: \mathscr{S}_{\infty} \rightarrow[0,1]$ as follows:

In the notation of Definition 3.3, if $\sum_{j \in \mathbf{K}} P_{E_{j}} \in \mathscr{S}\left(\mathscr{L}_{n}\right)$, then $\mu\left(\sum_{j \in K} P_{E_{j}}\right)=$ $2^{-n} \sharp(K)$, where $\sharp(K)$ is the cardinality of the set $K$. It is trivially easy to verify that $\mu$ is a well defined function because of the nested structure of $\mathscr{S}_{\infty}$.

Proposition 3.6. The function $\mu$ in Definition 3.5 is (finitely) additive and $\preceq$ continuous.

Proof. The additivity is obvious from the definition. To show the continuity, we first note that if $P_{j} \in \mathscr{S}_{\infty} \forall j$, then $P_{j} \lesseqgtr \mathbf{O} \Leftrightarrow \mu\left(P_{j}\right) \rightarrow 0$. Now let $P_{j}$ be a $\preceq$ increasing sequence in $\mathscr{S}_{\infty}$ which $\preceq$ converges to $P \in \mathscr{S}_{\infty}$. Because of the nesting in $\mathscr{S}_{\infty}$, we may assume without loss of generality that $P_{j} \leq P \forall j$. 
Thus $P-P_{j} \leftrightarrows \mathbb{O}$ and hence $\mu(\mathbb{P})=\lim \mu\left(P_{j}\right)$, by the above remark and by additivity. We similarly reach the same conclusion if $\mathbb{P}_{j}$ is a $\preceq$ decreasing sequence. Now let $\mu\left(\mathbb{P}_{j}\right)$ be any sequence in $\mathscr{S}_{\infty}$ which $\preceq$ converges to $\mathbb{P} \in \mathscr{S}_{\infty}$. If $\mu\left(P_{j}\right)$ does not converge to $\mu(\mathbb{P})$, then $P_{j}$ has either a $\preceq$ decreasing or a $\preceq$ increasing subsequence $P_{j_{k}}$ such that $\mu\left(P_{j_{k}}\right) f \mu(P)$, in contradiction with the results established above. This leads to the required result.

The continuity proved in Proposition 3.6 and the $\preceq$ density of $\mathscr{S}_{\infty}$ in $\mathscr{P}(\mathscr{H})$ allows us to extend $\mu$ to a $\preceq$ continuous function on all of $\mathscr{P}(\mathscr{H})$. Accordingly we regard the domain of $\mu$ to be all of $\mathscr{P}(\mathscr{H})$ from now on. Clearly $\mu$ satisfies $P \preceq Q \Leftrightarrow \mu(\mathbb{P}) \leq \mu(Q) \forall P, Q \in \mathscr{P}(\mathscr{H})$. It is clear that joint $\preceq$ continuity of addition would immediately imply additivity of $\mu$ on all of $\mathscr{P}(\mathscr{H})$ which, by Theorem 1.2, would lead to the implementation of $\preceq$ by a state. However, in view of Lemma 2.6 and Proposition 2.9, we have the following obvious result:

Lemma 3.7. Let $P_{E} \in \mathscr{D}(\mathscr{H})$. If $P_{E} \preceq \mathbb{P}_{E}^{\perp}$ then $\mu$ is additive on $\mathscr{P}(E)$.

Lemma 3.8. Let $S_{1}, S_{2} \in \mathscr{S}_{\infty}$ be mutually orthogonal. If $P_{1} \leq S_{1}$ and $P_{2} \leq S_{2}$ then $\mu\left(P_{1}+P_{2}\right)=\mu\left(P_{1}\right)+\mu\left(P_{2}\right)$.

Proof. We first look at the case $P_{1} \in \mathscr{S}_{\infty}$. Now there clearly exists a sequence $Q_{j}$ in $\mathscr{S}_{\infty}$ such that $Q_{j} \leq S_{2}$ and such that $Q_{j} \stackrel{\lessgtr}{\rightarrow} \mathbb{P}_{2}$. Separate $\preceq$ continuity of addition, $\preceq$ continuity of $\mu$ and additivity of $\mu$ on $\mathscr{S}_{\infty}$ lead to $\mu\left(P_{1}+P_{2}\right)=\lim _{j \rightarrow \infty} \mu\left(P_{1}+Q_{j}\right)=\lim _{j \rightarrow \infty}\left\{\mu\left(P_{1}\right)+\mu\left(Q_{j}\right)\right\}=\mu\left(P_{1}\right)+\mu\left(P_{2}\right)$ as required. If $P_{1} \notin \mathscr{S}_{\infty}$, then we again have $\mu\left(\mathbb{P}_{1}+\mathbb{P}_{2}\right)=\lim _{j \rightarrow \infty} \mu\left(P_{1}+Q_{j}\right)=\lim _{j \rightarrow \infty}\left\{\mu\left(\mathbb{P}_{1}\right)+\mu\left(Q_{j}\right)\right\}$ $=\mu\left(P_{1}\right)+\mu\left(P_{2}\right)$, where we have used the additivity proved in the case $\mathbb{P}_{1} \in \mathscr{S}_{\infty}$. This completes the proof.

Lemma 3.9. Suppose we have another sequence of nested equipartitions $\tilde{\mathscr{L}}_{n}$ of the identity such that $\tilde{\mathscr{L}}_{n}$ is of order $2^{n}$. Let $\tilde{\mathscr{S}}_{\infty}=\bigcup_{n \in \mathbb{N}} \mathscr{S}\left(\tilde{\mathscr{L}}_{n}\right)$, in the notation of Definition 3.3. Suppose that for some $n \in \mathbb{N}$ there exist $\mathbb{P}_{E} \in \mathscr{L}_{n}$ and $\mathbb{P}_{\tilde{E}} \in \tilde{\mathscr{L}}_{n}$ such that $P_{E} \leq P_{\tilde{E}}$, then the following are true:

घ. $P_{E}=P_{\tilde{E}}$.

ii. If $\tilde{\mu}$ is the function constructed from $\tilde{\mathscr{S}}_{\infty}$, as set out in Definition 3.5, then $\tilde{\mu}=\mu$.

Proof. : Assume the hypothesis and suppose the result is not true so that $P_{E}<P_{\tilde{E}}$. Let $\mathscr{L}_{n}=\left\{P_{j}: j \in \mathbb{K}(n)\right\}$, where $\mathbb{P}_{1}=\mathbb{P}_{E}$ and $\tilde{\mathscr{L}}_{n}=\left\{\tilde{P}_{j}: j \in \mathbb{K}(n)\right\}$, where $\widetilde{P}_{1}=P_{\tilde{E}}$. Then for each $j \in \mathbb{K}(n)$, there exists $Q_{j} \in \mathscr{D}(\mathscr{H})$ such that $Q_{j} \cong P_{j}$ and $Q_{j}<\widetilde{P}_{j}$, with $Q_{1}=\mathbb{P}_{1}$. Define $\mathscr{A}$ to be $\left\{Q_{j}: j \in \mathbb{K}(n)\right\}$. We claim that $\mu\left(\sum_{k=1}^{2^{n}} Q_{k}\right)=\sum_{k=1}^{2^{n}} \mu\left(Q_{k}\right)$. Suppose inductively that $\mu\left(\sum_{Q \in \mathscr{B}} Q\right)=\sum_{Q \in \mathscr{B}} \mu(\mathcal{Q})$ for any 
subset $\mathscr{B}$ of $\mathscr{A}$ of cardinality $N$, where $1<N<2^{n}$. Now let $\left\{Q_{j_{k}}: 1 \leq k \leq\right.$ $N+1\}$ be any subset of $\mathscr{A}$ of cardinality $N+1$ such that $j_{k} \leq j_{k+1}$ for all k. The inductive hypothesis implies that $\sum_{k=2}^{N+1} \tilde{Q}_{j_{k}} \cong \sum_{k=2}^{N+1} P_{k}$ so that application of axiom $\mathbb{A} 4$ and additivity of $\mu$ on $\mathscr{S}_{\infty}$ yields $\mu\left(\sum_{k=1}^{N+1} Q_{j_{k}}\right)=\mu\left(Q_{j_{1}}+\sum_{k=2}^{N+1} Q_{j_{k}}\right)=$ $\mu\left(P_{1}+\sum_{k=2}^{N+1} Q_{j_{k}}\right)=\mu\left(P_{1}+\sum_{k=2}^{N+1} P_{k}\right)=\sum_{k=1}^{N+1} \mu\left(P_{k}\right)=\sum_{k=1}^{N+1} \mu\left(Q_{j_{k}}\right)$. We remark that this argument also demonstrates that $\mu$ is additive on any subset of $\mathscr{A}$ of cardinality 2 , and so verifying the claim by induction. Since $Q_{k} \cong P_{k} \forall k \in K(n)$, this additivity gives $\mu\left(\sum_{k=1}^{2^{n}} Q_{k}\right)=1$, contradicting $Q_{k} \prec \tilde{P}_{k} \forall k \in K(n)$. This completes proof.

ii. The proof of item (i) shows that $\mu$ is additive on $\tilde{\mathscr{L}}_{n}$. We now show that $\mu$ is also additive on $\tilde{\mathscr{L}}_{m}$ for any $m \in \mathbb{N}$. If $m>n$ then $\tilde{\mathscr{L}}_{m}$ induces an equipartition $\tilde{B}$ of $P_{E}$ and $\mathscr{L}_{m}$ induces equipartitions $\mathscr{B}$ of $P_{E}$ and $\mathscr{C}$ of $P_{E}^{\perp}$. As $P_{E} \preceq P_{E}^{\perp}$, Lemma 2.6 implies that $\tilde{\mathscr{B}} \cup \mathscr{C}$ is also an equipartition of the identity of order $2^{m}$. Application of item (i.) shows that $\mu$ is additive on $\tilde{B} \cup \mathscr{C}$ and another application shows additivity on $\tilde{\mathscr{L}}_{m}$ and hence on all of $\tilde{\mathscr{S}}_{\infty}$. This immediately gives $\mu=\tilde{\mu}$.

Proposition 3.10. Let $P_{E} \in \mathscr{S}_{\infty}$, then $\mu$ is additive on $\mathscr{P}(E)$.

Proof. Let $P, Q \in \mathscr{P}(E)$ be mutually orthogonal. We can clearly construct a set of nested equipartitions $\tilde{\mathscr{L}}_{n}$ of the identity such that there exist $\tilde{S} \in \tilde{\mathscr{S}}_{\infty}=$ $\bigcup_{n \in N} \mathscr{S}\left(\tilde{\mathscr{L}}_{n}\right)$ and $Q^{\prime} \in \mathscr{P}(\mathscr{H})$ satisfying $P \leq \tilde{S}$ and $Q \cong Q^{\prime} \leq \tilde{S}^{\perp}$. By Lemma 3.9, we can also easily ensure that $\tilde{\mathscr{S}}_{\infty}$ is such that $\tilde{\mu}=\mu$. Lemma 3.8 gives $\tilde{\mu}(P+Q)=\tilde{\mu}\left(P+Q^{\prime}\right)=\tilde{\mu}(P)+\tilde{\mu}\left(Q^{\prime}\right)=\tilde{\mu}(P)+\tilde{\mu}(Q)$ as required.

Corollary 3.11. Let $P_{E} \in \mathscr{D}(\mathscr{H})$ be such that $P_{F} \leq P_{E}$ for some $P_{F} \in \mathscr{S}_{\infty}$, then $\mu$ is additive on $\mathscr{P}(E)$.

Proof. If $P_{E} \prec \mathbb{1}$ then by Lemma 3.9, the problem reduces to that of Proposition 3.10. So we assume that $P_{E} \cong \mathbb{1}$. By enlarging $P_{E}$ where necessary, we may also assume that $P_{E}-P_{F} \in \mathscr{D}(\mathscr{H})$ and that $O \prec P_{E}-P_{F}$. By Lemma 2.3, there exists a sequence $P_{G_{J}}$ in $\mathscr{D}(\mathscr{H})$ (where, in the notation of Lemma 2.3, $P_{G_{j}}$ is of the form $P_{f_{t}\left(E^{\perp}+F\right)}$, and where $\left.P_{f_{1}\left(E^{\perp}\right)}=P_{E}-P_{F}, P_{f_{t}(F)}=P_{F} \forall t\right)$ such that, for all $j, P_{G_{J}} \prec P_{E}, P_{F} \leq P_{G_{J}}$ and such that $P_{G_{J}} \stackrel{u}{\rightarrow} P_{E}$. Now let $P, Q \in \mathscr{P}(E)$ be mutually orthogonal. We choose that the sequence $P_{G_{j}}$ also satisfies item (iii) of Lemma 2.3 so that there exist sequences $P_{j}$ and $Q_{j}$ such that, for each $j, P_{j} \perp Q_{j}, P_{j}+Q_{j} \leq G_{j}$ and such that $P_{j} \stackrel{u}{\rightarrow} P, Q_{j} \stackrel{\leftrightarrow}{\rightarrow} Q$. Since $P_{j}+Q_{j} \stackrel{u}{\rightarrow} P+Q$, and since, by Proposition 3.10, $\mu$ is additive on each $\mathscr{P}\left(G_{j}\right)$, the result follows at once. 
Corollary 3.12. Let $\tilde{\mathscr{L}}_{n}$ be another sequence of nested equipartitions of the identity as set out in Lemma 3.9 and let $\tilde{\mu}$ be the function on $\mathscr{P}(\mathscr{H})$ constructed from the $\tilde{\mathscr{L}}_{n}$. Then $\tilde{\mu}=\mu$.

Proof. Let $P_{\tilde{E}} \in \tilde{\mathscr{S}}_{\infty}$ and let $P_{E} \in \mathscr{S}_{\infty}$. Let $P_{G} \leq P_{E}$ and $P_{\tilde{G}} \leq P_{\tilde{E}}$ be mutually orthogonal (see Lemma 2.2). The following two cases are sufficient for the proof.

Case $1 \quad 0<P_{G}$ and $O<P_{\tilde{G}}$.

We assume without loss of generality that $P_{G}+P_{\tilde{G}} \prec \mathbb{}-P_{G}-P_{\tilde{G}}$ and hence, by Lemma 3.7 , we may also assume that $P_{G} \in \mathscr{L}_{n}$ and that $P_{\tilde{G}} \in \widetilde{\mathscr{L}}_{n}$ for some $n \in \mathbb{N}$. Another sequence $\mathscr{L}_{k}^{\prime}$ of nested equipartitions of the identity, with the associated function $\mu^{\prime}$, can be constructed such that by an appropriate choice of the equipartition $\mathscr{L}_{n}^{\prime}$, Lemma 3.9 gives $\mu=\mu^{\prime}=\tilde{\mu}$ as required.

Case $2 \quad \mathbb{O} \cong P_{\tilde{G}}$.

By Corollary $3.11, \mu$ is additive on $\mathscr{P}\left(G^{\perp}\right)$ and $\tilde{\mu}$ on $\mathscr{P}\left(\tilde{G}^{\perp}\right)$. Let $\mathscr{L}_{n}^{\prime}$ be a sequence of nested equipartitions of $P_{F}$, where $P_{F}=\mathbb{B}-P_{G}-\mathbb{P}_{\tilde{G}}$, such that $\mathscr{L}_{n}^{\prime}=\left\{P_{n 1}, P_{n 2}, \ldots, P_{n 2^{n}}\right\}$. Then $\left\{\mathscr{L}_{n}^{\prime \prime}: n \in \mathbb{N}\right\}$, where $\mathscr{L}_{n}^{\prime \prime}=\left\{\mathbb{P}_{n 1}+\mathbb{P}_{\tilde{G}}, \mathbb{P}_{n 2}, \mathbb{P}_{n 3}\right.$, $\left.\ldots, P_{n 2^{n}}\right\}$ is a sequence of nested equipartitions of $P_{\bar{G}}$. Since $\tilde{\mu}$ is additive on $\mathscr{P}(F), \tilde{\mu}=\mu$ on $\mathscr{P}\left(G^{\perp}\right)$. As $P_{E} \in \mathscr{S}_{\infty}$ is arbitrary, we have $\tilde{\mu}=\mu$ on $\mathscr{S}_{\infty}$, and hence on $\mathscr{P}(\mathscr{H})$.

Proposition 3.13. $\mu$ is an additive measure on $\mathscr{P}(\mathscr{H})$. If $\preceq$ is weakly continuous, then $\mu$ is completely additive.

Proof. Let $P_{F}, P_{G} \in \mathscr{P}(\mathscr{H})$ be mutually orthogonal. Clearly, one can construct a sequence of nested equipartitions of the identity such that if $\mu^{\prime}$ is the associated function, then $\mu^{\prime}(P+Q)=\mu^{\prime}(P)+\mu^{\prime}(Q)$. Corollary 3.12 gives the required additivity.

Now let $\preceq$ be weakly continuous and let $\mathscr{A}=\left\{P_{j}: j \in \Lambda\right\}$ be a set of mutually orthogonal projections of $\mathscr{P}(\mathscr{H})$. By Theorem 2.8 , the set $\mathscr{B}$ of projections $P \in \mathscr{A}$ satisfying $\mu(P) \neq 0$ is, at most, countably infinite. Additivity and weak continuity of $\mu$ ensure that $\sum_{P \in \mathscr{B}} \mu(P)=\mu\left(\sum_{P \in \mathscr{B}} P\right)$. Weak continuity also implies that $\mu\left(\sum_{P \in \mathscr{A} \backslash \mathscr{B}} P\right)=0$ (see [1], Proposition 2.4 (ii)); this leads to the required complete additivity.

Theorem 1.2 and Proposition 3.13 lead to the final result:

Theorem 3.14. Let $\mathscr{H}$ be an infinite dimensional (not necessarily separable) Hilbert space and let $\preceq$ be a $\mathrm{CP}$ on $\mathscr{P}(\mathscr{H})$. Then $\preceq$ can be implemented by a (unique) state $\mu_{\leq}$on $\mathscr{B}(\mathscr{H})$ if and only if $\preceq$ is uniformly continuous. If $\preceq$ is weakly continuous, then $\mu_{\leq}$is normal. 


\section{Acknowledgements}

The author would like to thank Professor Abdus Salam, the International Atomic Energy Agency, UNESCO and the Swedish Development Cooperation for their support and hospitality at the International Centre for Theoretical Physics, Trieste, Italy, where part of this work was done.

\section{References}

[1] Mutangadura S. A., Implementation of Comparative Probability by Normal States, Commun. Math. Phys., 132 (1990), 581-592.

[2] Maeda S., Probability Measures on Projections in Von Neumann Algebras, Reviews in Mathematical Physics, 1 (1990), 235-290.

[3] Gleason A. M., Measures on the Closed Subspaces of a Hilbert Space, J. Math. and Mech., 6 (1957), 885-893.

[4] Kalmbach G., Measures and Hilbert Lattices, World Scientific, 1986.

[5] Ochs W., Gleason Measures and Quantum Comparative Probability, Quantum Probability and Applications II (Heidelberg Proceedings), (1985), 388-396.

[6] Goldstein S. and Paszkiewicz A., Comparison of States and Darboux-Type Properties in Von Neumann Algebras, Mathematica Scandinavica, 63 (1988), 220-232.

[7] Bratteli O. and Robinson D. W., Operator Algebras and Quantum Statistical Mechanics I, Springer-Verlag, 1979.

[8] Gaal S. A., Point Set Topology, Academic Press, 1964.

[9] Reed M. and Simon B., Methods of Modern Mathematical Physics, 1, Academic Press, 1980.

[10] Kelly J. L., General Topology, Van Nostrand, 1955. 
